\section{Infrared Heating System Doubles the Line Speed}

T

he British company Smith \& McLau-

rin, a global manufacturer of self-adhesive label, ticket and tag materials, has succeeded in more than doubling the line speed on one of its paper coating lines by using a carbon infrared heating system from Heraeus Noblelight. In addition, it also improved quality by eliminating uneven adhesive substrate patterns caused when drying coloured adhesives directly after application.

The company Smith \& McLaurin sells material in roll form to printers and converters who produce finished label ticket and tag products for a wide variety of end users, including supermarkets, pharmacy chains, logistics companies and food manufacturers. A special coating material for labels and security envelopes finds application in those areas in which security is important. This requires a water-based adhesive to be applied to a high value stock before a laminate is applied to the adhesive. In some cases, the laminated prod- uct is re-passed through the adhesive station so that the laminated layer itself can carry a layer of surface adhesive. Previously, the adhesive was dried in a warm air oven. However, this sometimes caused problems when drying coloured adhesives, resulting in "spotting" or reticulation, which created an uneven and visible adhesive pattern on the substrate. In the end, it was necessary to slow down the line to around 20 metres/min. To solve the problem, Heraeus supplied a portable infrared module to allow tests to be carried out on site. These proved so successful that a $32.5 \mathrm{~kW}$ system equipped with five carbon medium wave emitters was installed immediately before the existing warm air heaters. The emitters are individually controlled to enable them to be switched on and off according to the coating weight.

For further information, please contact: Heraeus Noblelight GmbH, D-63450 Hanau, www.heraeus-noblelight.com

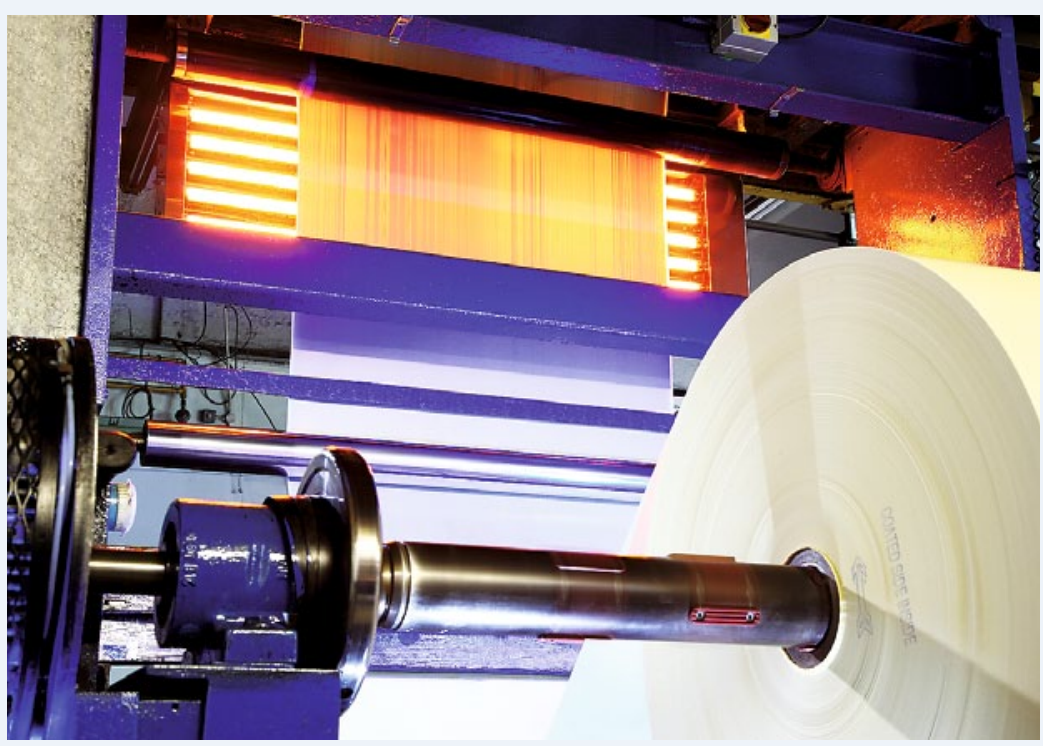

Infrared heating helps to more than double the line speed in the paper coating process (Source: Heraeus Noblelight)

\section{Sealant for Demanding Applications}

Q ealants for threaded joints on ma$\checkmark$ chines, devices and components which are used in the harshest environments, in which they are exposed to chemicals such as chlorine or powerful oxidisers such as oxygen, have to meet specific requirements. If anaerobic sealants are used in these circumstances, there is the risk of an explosion occurring.

A sealant produced by Hernon Manufacturing Inc., represented in Germany by 4 Advanced Technologies, which is chemically inert, non-flammable and non-toxic meets the required standards. It is hydrophobic, temperature resistant up to $450{ }^{\circ} \mathrm{F}$ or $232^{\circ} \mathrm{C}$ and is ideal for use in cryogenic and high-pressure applications (10,000 psi with 1/4" NPT threads). This product can also be used for sealing components that have to be repeatedly disassembled and assembled. Typical applications include oxygen and hydrogen systems, hydrogen peroxide and ozone systems, medical equipment, water treatment plants, paper processing, oil drilling and pumping facilities, plasma etching systems and welding machines. For more information, please contact: twitt@4advancedtechnologies.de 